\title{
ON AN OPEN QUESTION OF RICCERI CONCERNING A NEUMANN PROBLEM
}

\author{
FRANCESCA FARACI \\ University of Catania, Department of Mathematics, 95125 Catania, Italy \\ email: ffaraci@dmi.unict.it \\ and ALEXANDRU KRISTÁLY \\ University of Babeş-Bolyai, Department of Economics, 400591 Cluj-Napoca, Romania \\ email: alexandrukristaly@yahoo.com
}

(Received 13 January, 2006; accepted 30 November, 2006)

\begin{abstract}
In this paper we solve partially an open problem raised by B. Ricceri (Bull. London Math. Soc. 33 (2001), 331-340). Infinitely many solutions for a Neumann problem are obtained through a direct variational approach where the nonlinearity has an oscillatory behaviour at infinity.
\end{abstract}

2000 Mathematics Subject Classification. 35J20, 35J25.

1. Introduction. This paper is motivated by a problem raised by B. Ricceri in [4] (see also [5]) where the existence of infinitely many weak solutions for a Neumann problem has been proved under a highly oscillatory assumption on the nonlinearity. For the sake of clarity we recall the main result from [4] which led the author to formulate the open question we are dealing with.

Throughout the paper, $\Omega \subset \mathbb{R}^{N}$ is a bounded open set with smooth boundary, $v$ is the outer unit normal to $\partial \Omega, \lambda \in L^{\infty}(\Omega)$ with $\operatorname{essinf}_{\Omega} \lambda>0, \alpha \in L^{1}(\Omega)$ with $\alpha \geq 0$.

TheOREM 1 [4, Theorem 1]. Assume $p>N$. Let $f: \mathbb{R} \rightarrow \mathbb{R}$ be a continuous function, $\left\{r_{n}\right\} \subset \mathbb{R}^{+}$and $\left\{\xi_{n}\right\} \subset \mathbb{R}$ two sequences such that $\lim _{n \rightarrow \infty} r_{n}=+\infty$ and for each $n \in \mathbb{N}$, one has

$$
\frac{\left|\xi_{n}\right|^{p}}{p} \int_{\Omega} \lambda(x) d x<r_{n}
$$

and

$$
\int_{0}^{\xi_{n}} f(t) d t=\sup _{|\xi| \leq c\left(p r_{n}\right)^{\frac{1}{p}}} \int_{0}^{\xi} f(t) d t
$$

where

$$
c=\sup _{u \in W^{1, p}(\Omega) \backslash\{0\}} \frac{\sup _{x \in \Omega}|u(x)|}{\left(\int_{\Omega}|\nabla u(x)|^{p} d x+\int_{\Omega} \lambda(x)|u(x)|^{p} d x\right)^{1 / p}} .
$$


Finally, assume that

$$
\limsup _{|\xi| \rightarrow+\infty} \frac{\int_{\Omega} \alpha(x) d x \int_{0}^{\xi} f(t) d t}{|\xi|^{p}}>\frac{\int_{\Omega} \lambda(x) d x}{p} .
$$

Then, problem

$$
\begin{cases}-\triangle_{p} u+\lambda(x)|u|^{p-2} u=\alpha(x) f(u) & \text { in } \Omega \\ \partial u / \partial v=0 & \text { on } \partial \Omega\end{cases}
$$

admits an unbounded sequence of weak solutions in $W^{1, p}(\Omega)$.

In [4, Remark 2, p. 335] we read: “..., we observe that from condition (1) it follows that $\left|\xi_{n}\right|<c\left(p r_{n}\right)^{1 / p}$. This observation leads to the following open question: assume that all the assumptions, except for (1) and (2), of Theorem 1 hold, and suppose that there is a divergent sequence $\left\{b_{n}\right\}$ in $\mathbb{R}^{+}$such that, for each $n \in \mathbb{N}$, one has

$$
\int_{0}^{\xi_{n}} f(t) d t=\sup _{|\xi| \leq b_{n}} \int_{0}^{\xi} f(t) d t
$$

for some $\xi_{n}$ with $\left|\xi_{n}\right|<b_{n}$. Then, does the conclusion of Theorem 1 hold?"

In this paper we will give a partial answer to this question in the affirmative. Before doing this, note that in Theorem 1 Ricceri controlled the nonlinearity $f: \mathbb{R} \rightarrow \mathbb{R}$ on both the negative and the positive axis, cf. (2), applying a recent variational principle proved by himself, see [3]. Beside the direct approach, the advantage of our method consists of assuming a suitable oscillatory behaviour of the nonlinear term on either the positive or the negative axis, together with an additional technical condition, in order to obtain the same conclusion as in Theorem 1. More precisely, we may prove the following theorem.

TheOREM 2 (Oscillation at $+\infty$ ). Assume $p>N$. Let $f: \mathbb{R} \rightarrow \mathbb{R}$ be a continuous function, $\left\{b_{n}\right\}$ and $\left\{\xi_{n}\right\}$ sequences in $\mathbb{R}^{+}$with $\xi_{n}<b_{n}$ and $\lim _{n \rightarrow \infty} b_{n}=+\infty$ such that, for each $n \in \mathbb{N}$ one has

$$
\int_{0}^{\xi_{n}} f(t) d t=\sup _{0 \leq \xi \leq b_{n}} \int_{0}^{\xi} f(t) d t
$$

Assume that

$$
\limsup _{\xi \rightarrow+\infty} \frac{\int_{\Omega} \alpha(x) d x \int_{0}^{\xi} f(t) d t}{\xi^{p}}>\frac{\int_{\Omega} \lambda(x) d x}{p}
$$

and that there exists a non-degenerate interval $I \subset \mathbb{R}^{-}$such that $\left.f\right|_{I} \geq 0$. Then, the same conclusion as in Theorem 1 holds.

TheOREM 3 (Oscillation at $-\infty$ ). Assume $p>N$. Let $f: \mathbb{R} \rightarrow \mathbb{R}$ be a continuous function, $\left\{b_{n}\right\}$ and $\left\{\xi_{n}\right\}$ sequences in $\mathbb{R}^{-}$with $b_{n}<\xi_{n}$ and $\lim _{n \rightarrow \infty} b_{n}=-\infty$ such that, for each $n \in \mathbb{N}$ one has

$$
\int_{0}^{\xi_{n}} f(t) d t=\sup _{b_{n} \leq \xi \leq 0} \int_{0}^{\xi} f(t) d t
$$


Assume that

$$
\limsup _{\xi \rightarrow-\infty} \frac{\int_{\Omega} \alpha(x) d x \int_{0}^{\xi} f(t) d t}{|\xi|^{p}}>\frac{\int_{\Omega} \lambda(x) d x}{p}
$$

and that there exists a non-degenerate interval $I \subset \mathbb{R}^{+}$such that $\left.f\right|_{I} \leq 0$. Then, the same conclusion as in Theorem 1 holds.

REMARK 1. Note that Theorems 2 and 3 are equivalent in the sense that they are deducible from each other. Indeed, let $b_{n}^{\prime}:=-b_{n}, \xi_{n}^{\prime}:=-\xi_{n}, g(s)=-f(-s), s \in \mathbb{R}$, where $b_{n}, \xi_{n}, f$ fulfill the hypotheses of Theorem 3 . After an elementary calculation one can see that $b_{n}^{\prime}, \xi_{n}^{\prime}$, and $g$ verify all the assumptions of Theorem 2 . Thus, the problem

$$
\begin{cases}-\triangle_{p}(-u)+\lambda(x)|-u|^{p-2}(-u)=\alpha(x) f(-u) & \text { in } \Omega \\ \partial(-u) / \partial v=0 & \text { on } \partial \Omega\end{cases}
$$

admits an unbounded sequence of weak solutions in $W^{1, p}(\Omega)$, which concludes the argument.

REMARK 2. Comparing the hypotheses of Theorems 2 and 3 with the original problem raised by Ricceri, we mention the following differences:

1. We need not have full control of the nonlinearity $f$ on the whole real axis; compare (4) with $\left(4_{+}\right)$and $\left(4_{-}\right)$, respectively.

2. Conditions (5) and (6) are stronger than (3).

3. We need extra condition on $f$ on the other side of the real axis where the oscillatory behaviour is assumed.

REMARK 3. A similar question as we quoted earlier was formulated by Ricceri for the case when $\left\{b_{n}\right\}$ tends to zero. This problem has been partially solved by Anello and Cordaro in [1]. Note that in Anello and Cordaro's framework a suitable truncation of the nonlinearity can be employed, due to the convergence of $\left\{b_{n}\right\}$ to zero. Unfortunately, this technique fails in our context since $\left\{b_{n}\right\}$ diverges. This fact is compensated for in a certain sense by 3 . of Remark 2, which cannot be avoided in our argument. It would be interesting to prove/disprove that this condition can be removed.

Our approach is variational; weak solutions of $(P)$ will be obtained as local minima of the energy functional associated to $(P)$. To be more precise, let $W^{1, p}(\Omega)$ be endowed with the norm

$$
\|u\|=\left(\int_{\Omega}|\nabla u(x)|^{p} d x+\int_{\Omega} \lambda(x)|u(x)|^{p} d x\right)^{1 / p}
$$

which is equivalent to the standard norm in $W^{1, p}(\Omega)$. Let $F: \mathbb{R} \rightarrow \mathbb{R}$ be defined by $F(\xi)=\int_{0}^{\xi} f(t) d t$. The functional $\Phi: W^{1, p}(\Omega) \rightarrow \mathbb{R}$ defined by $\Phi(u)=$ $-\int_{\Omega} \alpha(x) F(u(x)) d x$ is sequentially weakly continuous on $W^{1, p}(\Omega)$ due to the compact embedding of $W^{1, p}(\Omega)$ into $C^{0}(\bar{\Omega})(p>N)$. (As usual $C^{0}(\bar{\Omega})$ is endowed with the sup-norm.) Moreover $\Phi$ is continuously Gâteaux differentiable with derivative given by $\Phi^{\prime}(u)(v)=-\int_{\Omega} \alpha(x) f(u(x)) v(x) d x$ for every $u, v \in W^{1, p}(\Omega)$. Thus, critical points (in particular, local minima) of the energy functional $\mathcal{E}(u) \stackrel{\text { def. }}{=} \frac{1}{p}\|u\|^{p}+\Phi(u)$ are weak solutions of problem $(P)$. 
In order to guarantee the existence of infinitely many local minima of $\mathcal{E}$ we construct a sequence of subsets in $C^{0}(\bar{\Omega})$ such that the relative minima of the energy $\mathcal{E}$ on these sets are actually local minima for the energy on $W^{1, p}(\Omega)$; this technique has been suggested by an idea of Saint Raymond [6].

2. Proof of Theorem 2. Let $\left\{b_{n}\right\}$ and $\left\{\xi_{n}\right\}$ be as in the statement of Theorem 2 . Notice that the sequence $\left\{\xi_{n}\right\}$ is unbounded; otherwise we would obtain a contradiction of (5). Thus, without loss of generality we may assume (up to subsequences) that $b_{n-1}<\xi_{n}<b_{n}$. By $\left(4_{+}\right)$, one can deduce the existence of a sequence $\left\{\xi_{n}^{\prime}\right\}$ in $\mathbb{R}^{+}$such that $\xi_{n}<\xi_{n}^{\prime}<b_{n}$ and

$$
F(\xi) \leq F\left(\xi_{n}\right), \quad \text { for all } \xi \in\left[\xi_{n}, \xi_{n}^{\prime}\right]
$$

In the same way, since there exists an interval $I \subset \mathbb{R}^{-}$such that $\left.f\right|_{I} \geq 0$, it is possible to find $\xi_{0}^{\prime}<\xi_{0}<0$ such that

$$
F(\xi) \leq F\left(\xi_{0}\right), \quad \text { for all } \xi \in\left[\xi_{0}^{\prime}, \xi_{0}\right]
$$

Define the set

$$
E_{n}=\left\{u \in W^{1, p}(\Omega): \xi_{0}^{\prime} \leq u(x) \leq \xi_{n}^{\prime} \text { for all } x \in \Omega\right\} .
$$

Claim 1. $\mathcal{E}$ is bounded from below on $E_{n}$ and its infimum on $E_{n}$ is attained.

It is clear that $E_{n}$ is convex. Moreover, it is closed in $W^{1, p}(\Omega)$ due to the continuity of the embedding $W^{1, p}(\Omega) \hookrightarrow C^{0}(\bar{\Omega})$; then $E_{n}$ is weakly closed. Since

$$
\mathcal{E}(u)=\frac{\|u\|^{p}}{p}-\int_{\Omega} \alpha(x) F(u) \geq-\|\alpha\|_{1} \max _{\left[\xi_{0}^{\prime}, \xi_{n}^{\prime}\right]} F \quad \text { for all } u \in E_{n},
$$

$\mathcal{E}$ is bounded from below on $E_{n}$. Let $\beta_{n}=\inf _{E_{n}} \mathcal{E}$, and $\left\{u_{k}\right\}$ a sequence in $E_{n}$ such that $\beta_{n} \leq \mathcal{E}\left(u_{k}\right) \leq \beta_{n}+1 / k$ for all $k \in \mathbb{N}$. Then,

$$
\left\|u_{k}\right\|^{p} / p \leq \beta_{n}+1+\|\alpha\|_{1} \max _{\left[\xi_{0}^{\prime}, \xi_{n}^{\prime}\right]} F
$$

for all $k \in \mathbb{N}$, i.e. $\left\{u_{k}\right\}$ is bounded in $W^{1, p}(\Omega)$. So, up to a subsequence, $\left\{u_{k}\right\}$ weakly converges in $W^{1, p}(\Omega)$ to some $\tilde{u}_{n} \in E_{n}$. By the sequentially weakly lower semicontinuity of $\mathcal{E}$ we conclude that $\mathcal{E}\left(\tilde{u}_{n}\right)=\beta_{n}=\inf _{E_{n}} \mathcal{E}$.

Claim 2. $\xi_{0} \leq \tilde{u}_{n}(x) \leq \xi_{n}$ for all $x \in \Omega$.

Let $X=\left\{x \in \Omega: \tilde{u}_{n}(x) \notin\left[\xi_{0}, \xi_{n}\right]\right\}$ and suppose that $X \neq \emptyset$. Thus, $m(X)>0$ (where $m(X)$ denotes the Lebesgue measure of $X)$, due to the continuity of $\tilde{u}_{n}$. Define

$$
h(\xi)= \begin{cases}\xi_{0}, & \text { if } \xi<\xi_{0} \\ \xi, & \text { if } \xi \in\left[\xi_{0}, \xi_{n}\right] \\ \xi_{n}, & \text { if } \xi>\xi_{n} .\end{cases}
$$

Set $\tilde{v}_{n}=h \circ \tilde{u}_{n}$. Due to Marcus and Mizel [2], $\tilde{v}_{n}$ belongs to $W^{1, p}(\Omega)$ (since $h$ is uniformly Lipschitz). Moreover $\tilde{v}_{n} \in E_{n}$. Denoting by

$$
X_{1}=\left\{x \in X: \tilde{u}_{n}(x)<\xi_{0}\right\} \quad \text { and } \quad X_{2}=\left\{x \in X: \tilde{u}_{n}(x)>\xi_{n}\right\},
$$


we have that $\tilde{v}_{n}(x)=\tilde{u}_{n}(x)$ for all $x \in \Omega \backslash X, \tilde{v}_{n}(x)=\xi_{0}$ for all $x \in X_{1}$ and $\tilde{v}_{n}(x)=\xi_{n}$ for all $x \in X_{2}$. Then,

$$
\begin{aligned}
\mathcal{E}\left(\tilde{v}_{n}\right)-\mathcal{E}\left(\tilde{u}_{n}\right)= & -\frac{1}{p} \int_{X}\left|\nabla \tilde{u}_{n}\right|^{p}+\frac{1}{p} \int_{X} \lambda(x)\left[\left|\tilde{v}_{n}\right|^{p}-\left|\tilde{u}_{n}\right|^{p}\right]-\int_{X} \alpha(x)\left[F\left(\tilde{v}_{n}\right)-F\left(\tilde{u}_{n}\right)\right] \\
= & -\frac{1}{p} \int_{X}\left|\nabla \tilde{u}_{n}\right|^{p}+\frac{1}{p} \int_{X_{1}} \lambda(x)\left[\left|\xi_{0}\right|^{p}-\left|\tilde{u}_{n}\right|^{p}\right]+\frac{1}{p} \int_{X_{2}} \lambda(x)\left[\xi_{n}^{p}-\tilde{u}_{n}^{p}\right] \\
& +\int_{X_{1}}-\alpha(x)\left[F\left(\xi_{0}\right)-F\left(\tilde{u}_{n}\right)\right]+\int_{X_{2}}-\alpha(x)\left[F\left(\xi_{n}\right)-F\left(\tilde{u}_{n}\right)\right] .
\end{aligned}
$$

From (7) and (8) we obtain that every term of the above expression is not positive. On the other hand, since $\mathcal{E}\left(\tilde{v}_{n}\right) \geq \mathcal{E}\left(\tilde{u}_{n}\right)=\inf _{E_{n}} \mathcal{E}$, then in particular,

$$
\begin{aligned}
\int_{X}\left|\nabla \tilde{u}_{n}\right|^{p} & =0 \\
\int_{X_{1}} \lambda(x)\left[\left|\xi_{0}\right|^{p}-\left|\tilde{u}_{n}\right|^{p}\right] & =\int_{X_{2}} \lambda(x)\left[\xi_{n}^{p}-\tilde{u}_{n}^{p}\right]=0 .
\end{aligned}
$$

From the first equality we deduce the existence of a positive measured subset $Y$ of $X$ and a constant $C$ such that $\tilde{u}_{n}=C$ on $Y$. Then, either $Y \subset X_{1}$ or $Y \subset X_{2}$. Assume that the first case occurs (analogously if $Y \subset X_{2}$ ). So,

$$
\begin{aligned}
0 & =\int_{X_{1}} \lambda(x)\left[\left|\xi_{0}\right|^{p}-\left|\tilde{u}_{n}\right|^{p}\right] \leq \int_{Y} \lambda(x)\left[\left|\xi_{0}\right|^{p}-|C|^{p}\right] \\
& \leq \operatorname{essinf}_{\Omega} \lambda\left[\left|\xi_{0}\right|^{p}-|C|^{p}\right] m(Y)<0
\end{aligned}
$$

a contradiction. This shows that $X$ has zero measure, therefore, $X=\emptyset$.

Claim 3. $\tilde{u}_{n}$ is a local minimum of $\mathcal{E}$ in $W^{1, p}(\Omega)$.

Suppose the contrary. Then there exists a sequence $\left\{u_{k}\right\} \subset W^{1, p}(\Omega)$ such that it converges to $\tilde{u}_{n}$ and $\mathcal{E}\left(u_{k}\right)<\mathcal{E}\left(\tilde{u}_{n}\right)$ for all $k \in \mathbb{N}$. From the latter inequality, it follows that $u_{k} \notin E_{n}$ for any $k \in \mathbb{N}$. Since $u_{k} \rightarrow \tilde{u}_{n}$ in $W^{1, p}(\Omega)$, then $u_{k} \rightarrow \tilde{u}_{n}$ in $C^{0}(\bar{\Omega})$. In particular, for every $0<\varepsilon<\min \left\{\xi_{n}^{\prime}-\xi_{n}, \xi_{0}-\xi_{0}^{\prime}\right\} / 2$, there exists $k_{\varepsilon} \in \mathbb{N}$ such that $\sup _{x \in \Omega}\left|u_{k}(x)-\tilde{u}_{n}(x)\right|<\varepsilon$ for every $k \geq k_{\varepsilon}$. Taking into account the choice of the number $\varepsilon$, and using Claim 2 we conclude that

$$
\xi_{0}^{\prime}<u_{k}(x)<\xi_{n}^{\prime} \text { for all } x \in \Omega, k \geq k_{\varepsilon}
$$

which clearly contradicts the fact $u_{k} \notin E_{n}$.

Claim 4. $\lim _{n \rightarrow \infty} \beta_{n}=-\infty$. (Recall that $\beta_{n}=\inf _{E_{n}} \mathcal{E}$.)

From (5) there exist a sequence $\left\{\tilde{\xi}_{k}\right\} \subset \mathbb{R}^{+}$tending to $+\infty$ and a constant $M>0$ such that

$$
\frac{F\left(\tilde{\xi}_{k}\right) \int_{\Omega} \alpha(x) d x}{\tilde{\xi}_{k}^{p}}>M>\frac{\int_{\Omega} \lambda(x) d x}{p} .
$$


Since $\xi_{n}$ tends to $+\infty$, there exist a subsequence $\left\{\xi_{n_{k}}\right\}$ of $\left\{\xi_{n}\right\}$ and $\bar{k} \in \mathbb{N}$ such that $\tilde{\xi}_{k}<\xi_{n_{k}}$, for $k \geq \bar{k}$. Then, the constant function $w_{k}=\tilde{\xi}_{k}$ belongs to $E_{n_{k}}$ and

$$
\begin{aligned}
\beta_{n_{k}}=\inf _{E_{n_{k}}} \mathcal{E} \leq \mathcal{E}\left(w_{k}\right) & =\frac{\left\|w_{k}\right\|^{p}}{p}-F\left(\tilde{\xi}_{k}\right) \int_{\Omega} \alpha(x) d x \leq \frac{1}{p} \tilde{\xi}_{k}^{p} \int_{\Omega} \lambda(x) d x-M \tilde{\xi}_{k}^{p} \\
& =\tilde{\xi}_{k}^{p}\left(\frac{1}{p} \int_{\Omega} \lambda(x) d x-M\right) \rightarrow-\infty .
\end{aligned}
$$

Since $\left\{\beta_{n}\right\}$ is non-increasing, our claim is achieved.

Proof of Theorem 2 concluded. Since $\tilde{u}_{n}$ are local minima of $\mathcal{E}$ (cf. Claim 3), they are critical points of $\mathcal{E}$, thus weak solutions of $(P)$. Due to Claim 4 there are infinitely many pairwise distinct $\tilde{u}_{n}$. Moreover, one has $\left\|\tilde{u}_{n}\right\| \rightarrow \infty$ as $n \rightarrow \infty$. Indeed, arguing by contradiction, there is a subsequence $\left\{\tilde{u}_{n_{k}}\right\}$ of $\left\{\tilde{u}_{n}\right\}$ which is bounded in $W^{1, p}(\Omega)$. Thus, it is bounded in $C^{0}(\bar{\Omega})$ as well. In particular we can find $n_{0} \in \mathbb{N}$ such that $\tilde{u}_{n_{k}} \in E_{n_{0}}$ for every $k \in \mathbb{N}$. For every $n_{k} \geq n_{0}$ one has

$$
\beta_{n_{0}} \geq \beta_{n_{k}}=\inf _{E_{n_{k}}} \mathcal{E}=\mathcal{E}\left(\tilde{u}_{n_{k}}\right) \geq \inf _{E_{n_{0}}} \mathcal{E}=\beta_{n_{0}},
$$

which proves that $\beta_{n_{k}}=\beta_{n_{0}}$ for all $n_{k} \geq n_{0}$, contradicting Claim 4 .

3. Consequences, examples. In the sequel, we assume $p>N$, and $\alpha, \lambda$ are as in Section 1. The next result gives a simple criterion for applying Theorem 2.

COROllary 1 . Let $f: \mathbb{R} \rightarrow \mathbb{R}$ be a continuous function which fulfills (5) and let $I \subset \mathbb{R}^{-}$a non-degenerate interval such that $\left.f\right|_{I} \geq 0$. Assume that there are sequences $\left\{a_{n}\right\}$ and $\left\{b_{n}\right\}$ in $\mathbb{R}^{+}$with $a_{n}<b_{n}$ and $\lim _{n \rightarrow \infty} b_{n}=+\infty$ such that, for every $n \in \mathbb{N}$ one has

$$
f(t) \leq 0 \quad \text { for all } t \in\left[a_{n}, b_{n}\right]
$$

Then, problem $(P)$ admits an unbounded sequence of weak solutions in $W^{1, p}(\Omega)$. In particular, if $f \geq 0$ on $\mathbb{R}^{-}$, the solutions are non-negative.

Proof. By condition (9), one has $\int_{0}^{\xi} f(t) d t \leq \int_{0}^{a_{n}} f(t) d t$ for all $\xi \in\left[a_{n}, b_{n}\right]$. Hence, there exists a point $\left.\xi_{n} \in\right] 0, a_{n}$ ] such that condition $\left(4_{+}\right)$is verified. Now, we can apply Theorem 2.

When $f \geq 0$ on $\mathbb{R}^{-}$, the solutions are non-negative. Indeed, suppose that $u \in$ $W^{1, p}(\Omega)$ is a weak solution of $(P)$ and the set $S=\{x \in \Omega: u(x)<0\}$ is not empty. It is clear that $S$ is open. Let $u_{S} \in W^{1, p}(\Omega)$ be defined by $u_{S}=\min \{u, 0\}$. Then we obtain

$$
\int_{\Omega}\left(|\nabla u|^{p-2} \nabla u \nabla u_{S}+\lambda(x)|u|^{p-2} u u_{S}\right)=\int_{\Omega} \alpha(x) f(u) u_{S} .
$$

Using the above relation and the fact that $f \geq 0$ in $\mathbb{R}^{-}$, we conclude that $\|u\|_{W^{1, p}(S)}^{p}=$ $\int_{S}\left(|\nabla u|^{p}+\lambda(x)|u|^{p}\right)=\int_{S} \alpha(x) f(u) u \leq 0$, which contradicts the choice of the set $S$. This completes the proof.

REMARK 4. A similar result to Corollary 1 can be stated in view of Theorem 3. These kinds of results solve partially Problem 8 in [5]. Indeed, we can avoid in [4, Theorem 3] the condition $\lim _{n \rightarrow \infty} \frac{a_{n}}{b_{n}}=0$ which was essential in Ricceri's approach 
(see e.g. examples below). Note that Theorem 3 in [4] is a direct consequence of Theorem 1.

EXAMPLE 1. Let $p>N$ and $\lambda \in L^{\infty}(\Omega)$ with $\operatorname{essinf}_{\Omega} \lambda>0$. Let $\sigma>8$ and $A_{n}=$ $[2 n, 2 n+1]$ for every $n \in \mathbb{N}$. Then, the problem

$$
\begin{cases}-\triangle_{p} u=\lambda(x)|u|^{p-2} u\left[\sigma \operatorname{dist}\left(u, \mathbb{R} \backslash \cup_{n \in \mathbb{N}} A_{n}\right)-1\right] & \text { in } \Omega \\ \partial u / \partial v=0 & \text { on } \partial \Omega\end{cases}
$$

admits an unbounded sequence of non-negative weak solutions in $W^{1, p}(\Omega)$.

Proof. We take $f(t)=\sigma|t|^{p-2} t \operatorname{dist}\left(t, \mathbb{R} \backslash \cup_{n \in \mathbb{N}} A_{n}\right)$ and $\alpha(x)=\lambda(x), \quad a_{n}=$ $2 n+1, b_{n}=2 n+2$. Since $f \equiv 0$ in $\mathbb{R}^{-}$and $\lim \sup _{\xi \rightarrow+\infty} \frac{\int_{0}^{\xi} f(t) d t}{\xi^{p}} \geq \frac{\sigma}{8 p}$, we can apply Corollary 1 .

In the next example we denote by $[p]$ the integer part of $p \in \mathbb{R}$.

EXAMPLE 2. Let $p>N, \lambda \in L^{\infty}(\Omega)$ with $\operatorname{essinf}_{\Omega} \lambda>0$ and $\alpha \in L^{1}(\Omega) \backslash\{0\}$ with $\alpha \geq 0$. Then, the problem

$$
\begin{cases}-\triangle_{p} u+\lambda(x)|u|^{p-2} u=\alpha(x)|u|^{[p]+1} \sin u & \text { in } \Omega \\ \partial u / \partial v=0 & \text { on } \partial \Omega\end{cases}
$$

admits an unbounded sequence of weak solutions in $W^{1, p}(\Omega)$.

Proof. We apply Corollary 1 , taking $f(t)=|t|^{[p]+1} \sin t$ and $a_{n}=(2 n+1) \pi, b_{n}=$ $2(n+1) \pi$. Notice that $\lim \sup _{\xi \rightarrow+\infty} \frac{\int_{0}^{\xi} f(t) d t}{\xi^{p}}=+\infty$, thus relation $(5)$ is verified.

ACKNOwLEDGMENTS. This work has been initiated when Alexandru Kristály visited the Department of Mathematics of the University of Catania, Italy, in JuneJuly, 2005. He wishes to express his gratitude to Professor Biagio Ricceri for his kind invitation and hospitality. He was supported by the the CNCSIS project No.AT/870 and by the Istuto Nazionale di Alta Matematica (INDAM), Italy.

\section{REFERENCES}

1. G. Anello and G. Cordaro, Infinitely many positive solutions for the Neumann problem involving the $p$-Laplacian, Colloq. Math. 97 (2003), 221-231.

2. M. Marcus and V. Mizel, Every superposition operator mapping one Sobolev space into another is continuous, J. Functional Analysis 33 (1979), 217-229.

3. B. Ricceri, A general variational principle and some of its applications, J. Comput. Appl. Math. 113 (2000), 401-410.

4. B. Ricceri, Infinitely many solutions of the Neumann problem for elliptic equations involving the $p$-Laplacian, Bull. London Math. Soc. 33 (2001), 331-340.

5. B. Ricceri, Some research perspectives in nonlinear functional analysis, International Conference on Nonlinear Operators, Differential Equations and Applications (Cluj-Napoca, 2001) in Semin. Fixed Point Theory Cluj-Napoca 3 (2002), 99-109.

6. J. Saint Raymond, On the multiplicity of solutions of the equation $-\Delta u=\lambda f(u)$, J. Differential Equations 180 (2002), 65-88. 